\title{
Eosinophilic annular erythema in adults: report of two cases and review of the literature*
}

\author{
Marta Olivé Heras ${ }^{1}$ \\ Maribel Iglesias Sancho ${ }^{1}$
}

\author{
Noelia Pérez Muñoz ${ }^{2}$ \\ Pablo Umbert Millet ${ }^{1}$
}

DOI: http:/ /dx.doi.org/10.1590/abd1806-4841.20176373

\begin{abstract}
We herein report two cases of eosinophilic annular erythema in adults, which is rare. In both patients, lesions developed rapidly in few days and were located mainly on the trunk, buttocks, and extremities. Diagnosis was histopathological, with typical features including acute dermal inflammatory infiltrate with abundant eosinophils. One of the patients recurred after treatment on three occasions and finally cured spontaneously. The second patient recurred once and was then successfully treated with topical clobetasol. Clinical and histopathological features of eosinophilic annular erythema in adults have rarely been reported. A review of the literature and discussion of relationship with Wells syndrome are also included.
\end{abstract}

Keywords: Cellulitis; Clobetasol; Eosinophils; Erythema; Steroids

\section{INTRODUCTION}

Eosinophilic annular erythema (EAE) is an uncommon benign acute eosinophilic dermatosis of unknown etiology, originally described in children. ${ }^{1,2}$ EAE is clinically characterized by recurrent annular, erythematous-edematous, pruritic lesions with a centrifugal growth pattern. A limited number of EAE cases in adolescents and adults have been reported to date. ${ }^{3,4}$ We herein add two new adult cases of this rare entity, which shared clinical and histopathologic features that enabled this diagnosis. We also provide insight in the understanding of its possible relationship with Wells syndrome.

\section{CASE REPORT}

Case 1. A 74-year-old man presented with an annular lesion, on the lateral aspect of the thorax, which appeared two weeks prior to the consultation. Its growth was centrifugal with erythematous-edematous margins, and a minimally scaly center (Figure 1).
At the moment, a punch-biopsy was performed. Histopathological examination showed a perivascular and interstitial mixed infiltrate with abundant eosinophils, but without evidence of flame figures (Figure 2). Direct immunofluorescence test was negative. With the suspicion of Lyme disease, treatment with doxycycline was initiated. Laboratory tests including complete blood count, C-reactive protein, erythrocyte sedimentation rate, renal and liver function and Borrelia burgdorferi's serology were unrevealing.

The patient underwent three recurrences that were treated with topical clobetasol propionate cream. Three months later, a further recurrence healed spontaneously.

Case 2. A 75-year-old man presented with migrating, minimally scaly, annular lesions located on the trunk, buttocks, and limbs (Figure 3). Routine laboratory tests did not disclose any abnormality and included complete blood count, C-reactive protein,

Study submitted in 16.08.2016

Approved by the Advisory Board and accepted for publication on 27.11.2016

* Study conducted at the Hospital Universitari Sagrat Cor - Barcelona, Spain. Financial Support: None.

Conflict of Interests: None.

Department of Dermatology, Hospital Sagrat Cor - Barcelona, Spain.

Department of Pathological Anatomy, Hospital General de Catalunya - Barcelona, Spain.

@ 2017 by Anais Brasileiros de Dermatologia 
erythrocyte sedimentation rate, renal and liver function. A biopsy was taken and the histopathological examination revealed a chronic perivascular and interstitial inflammatory infiltrate with moderate amount of eosinophils in the superficial as well as in the deep dermis (Figure 4). Flame figures were absent. Direct immunofluorescence test was negative. The patient, with the clinical orientation of eosinophilic dermatosis, was treated with oral corticosteroids. Six months later, there was a recurrence, which was successfully treated with topical clobetasol, with complete clearance of the plaques.

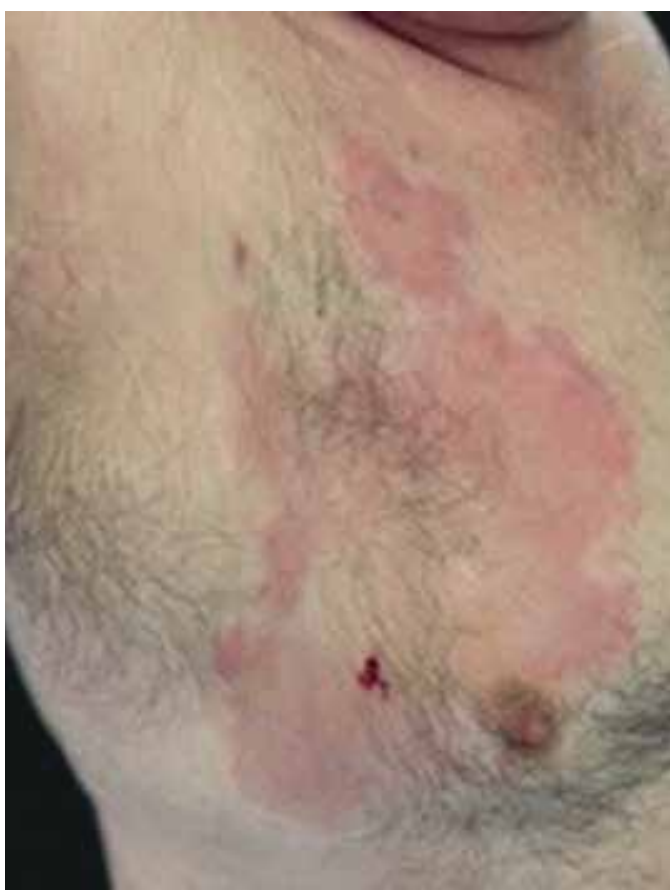

Figure 1: An annular lesion on one side of the thorax with erythematous-edematous margins, and a minimally scaly center
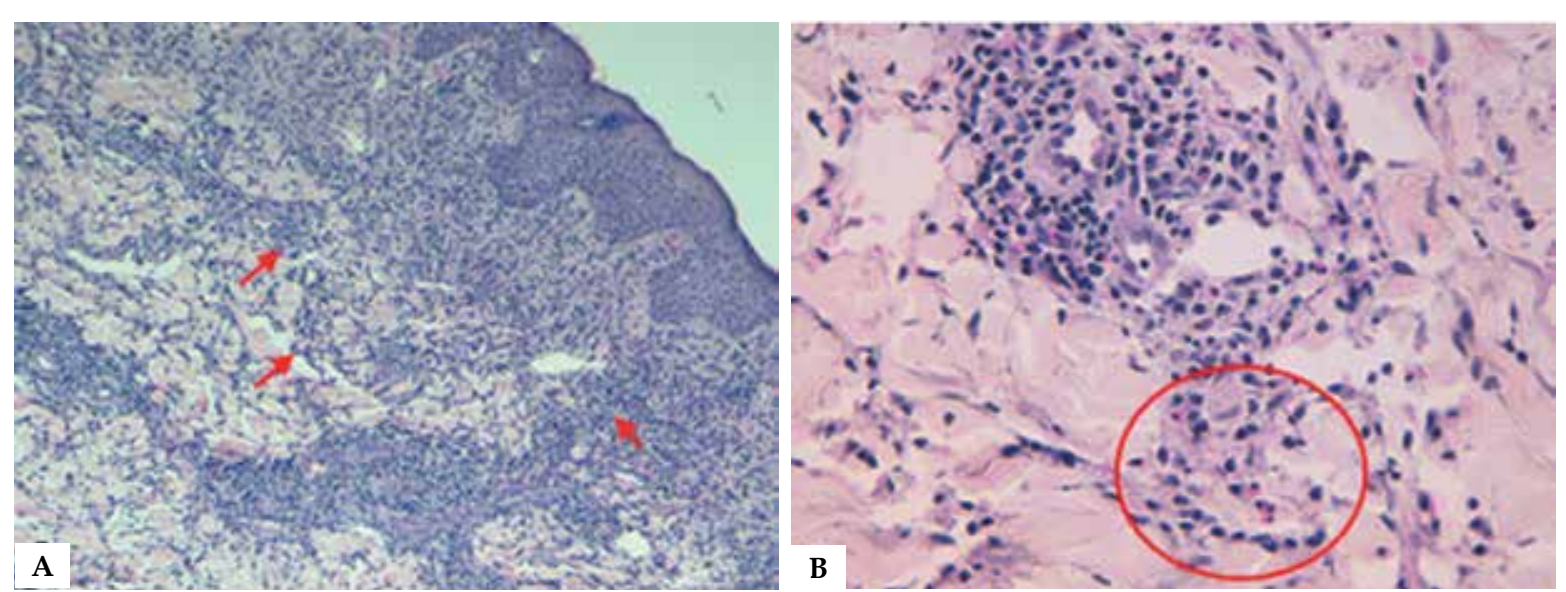

Figure 2: A: Hyperplasia of the epidermis. Perivascular and interstitial (arrow) mixed infiltrate with abundant eosinophils. Absence of flame figures and vasculitis (Hematoxylin \& eosin, X10). B: A cluster of eosinophils (circle), more abundant around the central vessel (Hematoxylin \& eosin, X40)

\section{DISCUSSION}

EAE is a benign eosinophilic dermatosis with excellent prognosis. The first description by Peterson and Jarratt in 1981 included only pediatric patients. ${ }^{5}$ Thus, it was initially named annular erythema of infancy. Children fulfilling the author's diagnostic criteria of EAE, usually healthy, showed slowly expanding arcuate or urticaria-like lesions. Skin manifestations were cyclic and persisted for some days up to weeks or months. Histologically, the authors described a perivascular lymphohistiocytic infiltrate with a prominent eosinophilic component and no alteration at the epidermic level. ${ }^{5}$

The first adult case of EAE was described by Kahofer et al. in $2000 .{ }^{6}$ They found similarities with the previously described EAE in children. Therefore, they decided to treat the patient with hydroxychloroquine, with optimal response.

In further published cases in following years, a possible association of EAE with autoimmune thyroid disease, chronic borreliosis, and renal cell carcinoma has been suggested. ${ }^{6,7}$ In most adult patients, regarding clinical manifestations, EAE presents as recurrent annular papules that evolve into palpable erythematous arches or rings.

Microscopic findings of the reviewed literature are identical for adult and pediatric patients. Proposed clinical differential diagnoses included: Wells syndrome, erythema chronicum migrans, erythema annulare centrifugum, erythema gyratum repens, and pemphigoid. After hematoxylin and eosin staining, histopathological pattern was only compatible with Wells syndrome or eosinophilic cellulitis, but a final diagnosis of EAE was achieved. Wells syndrome is histologically defined by an interstitial infiltrate of eosinophils, with lymphocytes and histiocytes. The infiltrate is usually more impressive in the deep dermis. Eosinophilic-staining flame figures consisting of collagen fibers coated with eosinophil granule proteins are a hallmark of this entity. In our patients diffuse inflammatory infiltrate in the dermis with abundant eosinophils was observed, but neither the so-called flame figures nor the vesicular change were present.

The major series of EAE cases, to our knowledge, corresponds to a multicentric study including 10 patients that was published in 2013 by El-Khalawany et al. ${ }^{8}$ The authors described typical 

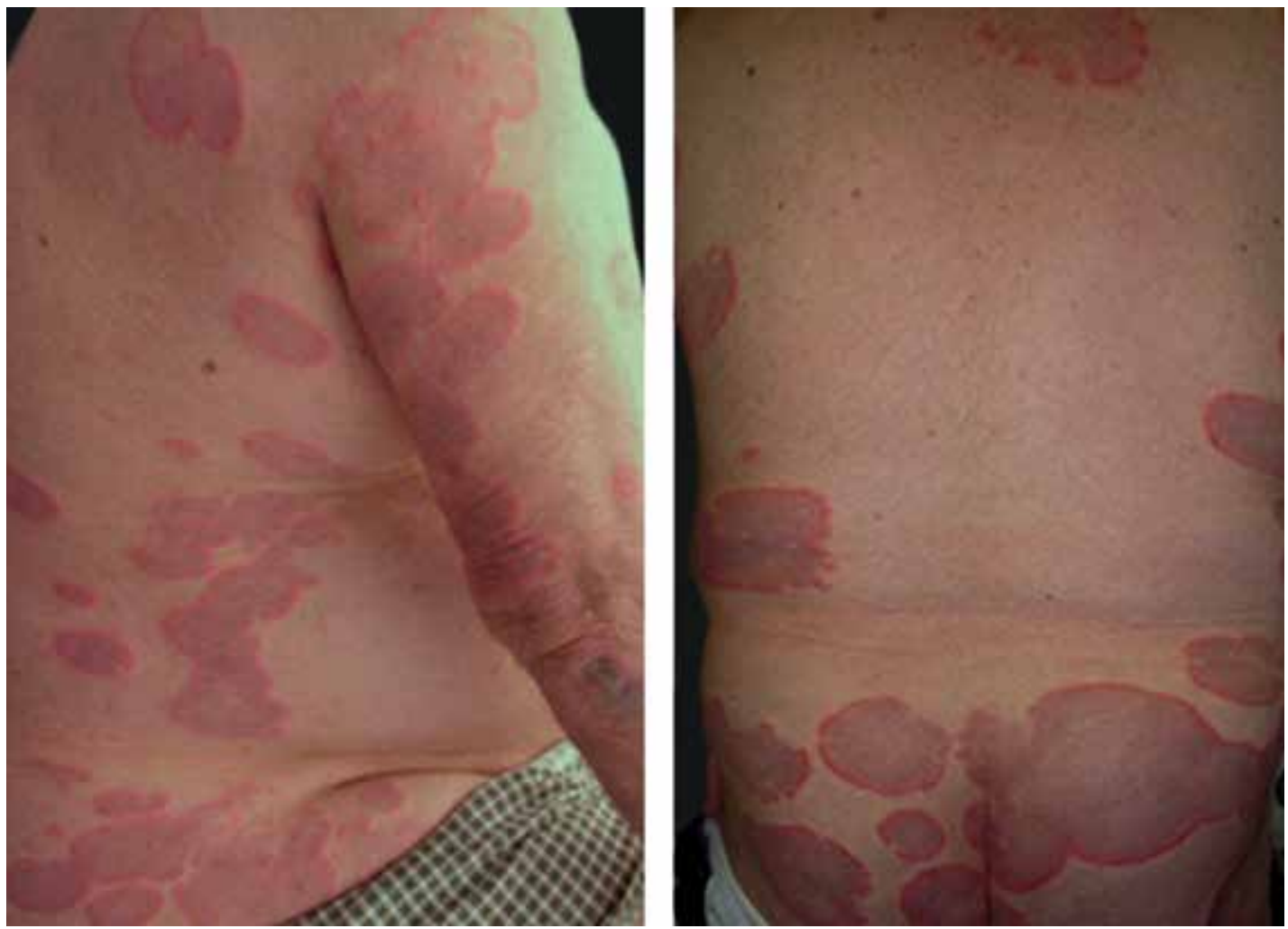

Figure 3:

Minimally scaly, annular, erythematous plaques on the buttocks and trunk
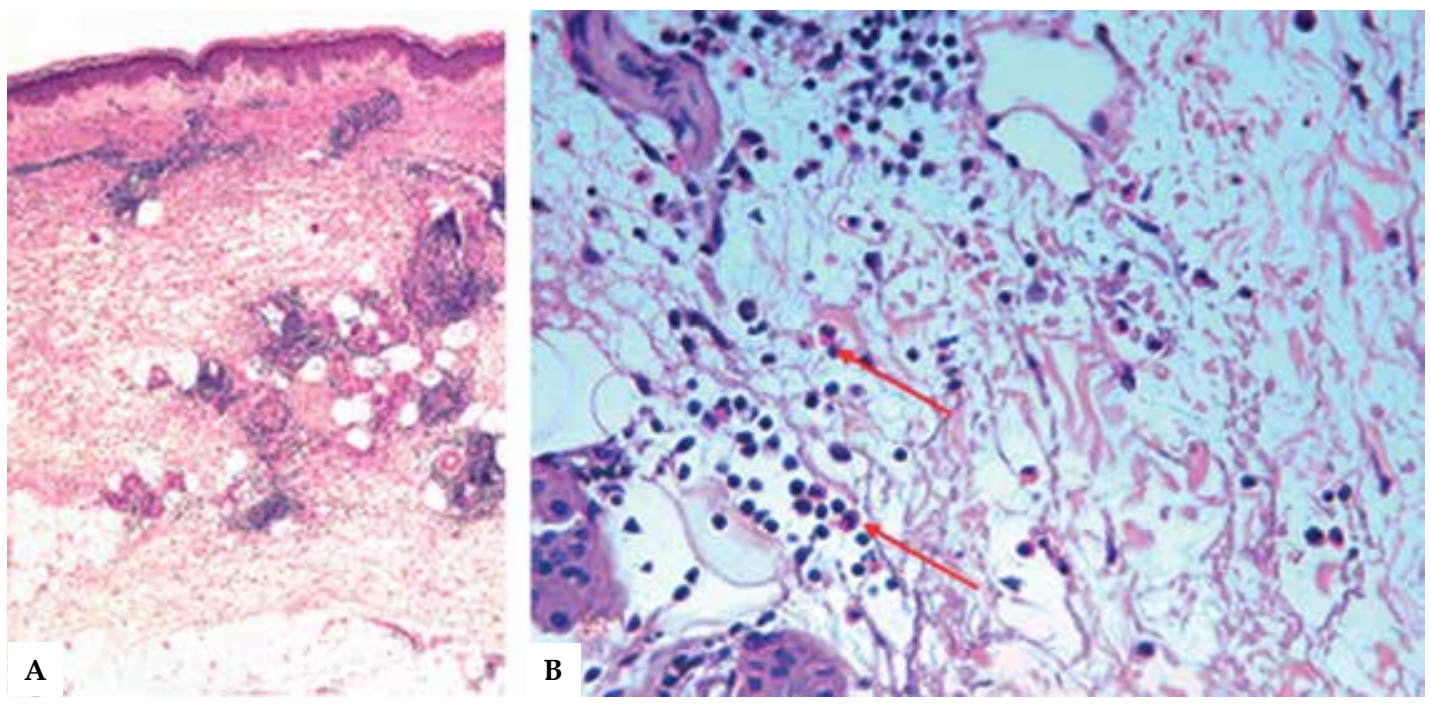

FIgURE 4: A: Superficial and deep dermatitis, perivascular and extending to the interstitium with presence of lymphocytes and plasma cells, as well as eosinophils. (Hematoxylin \& eosin, X8).

B: Presence of eosinophils (arrow) in the interstitium and inside a blood vessel (Hematoxylin \& eosin, $\mathrm{X} 40$ )

clinical presentation very similar to ours, and also included histopathological study. In the article some histopathological differential features seemed to correlate with the age of the lesions. Presence of flame figures and mucin deposition was observed in some instances. Those patients had a chronic course and were resistant to treatment with systemic corticosteroids alone or in combination with hydroxychloroquine and cyclosporine. The authors, therefore, concluded that EAE was a peculiar clinical variant in the spectrum of Wells syndrome. It is our understanding that maybe the worse response to the treatment in some of these patients corresponded to underdiagnosed Wells syndrome and not EAE with well-defined criteria.
Regarding biochemical tests, blood eosinophilia and high serum levels of interleukin 5 can be found in patients with Wells syndrome. ${ }^{9}$ As we already stated, no blood eosinophilia was found in our two patients. Some authors defend that peripheral blood eosinophilia and absence of response to antimalarial drugs would be two distinctive aspects of Wells syndrome when compared with EAE. ${ }^{5}$ Finally, EAE as well as Wells syndrome can present recurrences and spontaneous resolution after some months or years.

Regarding the clinical course, rapid disappearance of lesions of EAE with systemic corticoids is expected in most cases, although other authors have failed to find such a good response. ${ }^{4,10}$ In one of our patients, lesions recurred after discontinuation of 
corticosteroids but clobetasol cream was used for the treatment of recurrences with good response. In some cases reported in the literature, lesions cleared with antimalarial drugs. ${ }^{6,7}$ Other authors have also suggested the combination of oral prednisone and chloroquine or cyclosporine as possible alternatives. ${ }^{7,8}$

In summary, although some authors regard EAE as a variant of Wells syndrome, we believe that some subtle differences al- low this differential diagnosis. Despite the clinical similarity of both diseases, in EAE the inflammatory infiltrate is mainly perivascular and no flame figures are observed. Peripheral eosinophilia is absent, better pharmacological response might be expected and full recovery is obtained. $\square$

\section{REFERENCES}

1. Peterson AO Jr, Jarratt M. Annular erythema of infancy. Arch Dermatol. 1981;117:145-8.

2. Thomas L, Fatah S, Nagarajan S, Natarajan S. Eosinophilic annular erythema: successful response to ultraviolet B therapy. Clin Exp Dermatol. 2015;40:883-6.

3. Sempau L, Larralde M, Luna PC, Casas J, Staiger H. Eosinophilic anular erythema. Dermatol Online J. 2012:18:8.

4. Howes R, Girgis L, Kossard S. Eosinophilic annular erythema: a subset of Wells syndrome or a distinct entity? Australas J Dermatol. 2008:49:159-63.

5. López-Pestaña A, Tuneu A, Lobo C, Zubizarreta J, Eguino P. Eritema anular eosinofilico. Actas Dermosifilogr 2004:95:302-4.

6. Kahofer P, Grabmaier E, Aberer E. Treatment of eosinophilic annular erythema with chloroquine. Acta Derm Venereol. 2000;80:70-1.

7. Mebazaa A, Kenani N, Ghariani N, Denguezli M, Sriha B, Belajouza C, et al. Eosinophilic annular erythema responsive to chloroquin. Eur $\mathrm{J}$ Dermatol. 2009:19:84-5

8. El-Khalawany M, Al-Mutairi N, Sultan M, Shaaban D. Eosinophilic annular erythema is a peculiar subtype in the spectrum of Wells syndrome: a multicentre long-term follow-up study. J Eur Acad Dermatol Venereol. 2013;27:973-9.

9. España A, Sanz ML, Sola J, Gil P. Wells' syndrome (eosinophilic cellulitis) correlation between clinical activity, eosinophil levels, eosinophil cation protein and interleukin-5. Br J Dermatol. 1999;140:127-30.

10. Sinno H, Lacroix JP, Lee J, Izadpanah A, Borsuk R, Watters K, et al. Diagnosis and management of eosinophilic cellulitis (Wells' syndrome): A case series and literature review. Can J Plast Surg. 2012;20:91-7.
MAILING ADDRESS:

Marta Olivé Heras

Hospital Universitari Sagrat Cor

C/ Viladomat 288

08029 Barcelona, Spain.

Email: marta_olive_1987@hotmail.com

How to cite this article: Heras MO, Muñoz NP, Sancho MI, Millet PU. Eosinophilic annular erythema in adults: report of two cases and review of the literature. An Bras Dermatol. 2017;92(5 Suppl 1): 65-8. 